\title{
Testable implications of general equilibrium theory: A differentiable approach
}

Chiappori, Pierre-Andre ; Ekeland, I ; Kübler, Felix ; Polemarchakis, Herakles M

\begin{abstract}
Is general equilibrium theory empirically testable? Our perspective on this question differs fromthe standard, Sonnenschein-Debreu-Mantel (SDM) viewpoint. While the SDM tradition considersaggregate (excess) demand as a function of prices, we suppose that what is observable is the equilibriumprice vector as a function of the fundamentals of the economy. We apply this perspective to anexchange economy where equilibrium prices and individual endowments are observable. We derivenecessary and sufficient conditions that characterize the equilibrium prices, as functions of initialendowments. Furthermore, we show that, if these conditions are satisfied, then the economy cangenerically be identified. Finally, we show that when only aggregate data are available, observablerestrictions vanish. We conclude that the availability of individual data is essential for the derivationof testable consequences of the general equilibrium construct.
\end{abstract}

DOI: https://doi.org/10.1016/j.jmateco.2003.11.002

Posted at the Zurich Open Repository and Archive, University of Zurich

ZORA URL: https://doi.org/10.5167/uzh-62195

Journal Article

Accepted Version

Originally published at:

Chiappori, Pierre-Andre; Ekeland, I; Kübler, Felix; Polemarchakis, Herakles M (2004). Testable implications of general equilibrium theory: A differentiable approach. Journal of Mathematical Economics, 40(1-2):105-119.

DOI: https://doi.org/10.1016/j.jmateco.2003.11.002 


\title{
Testable implications of general equilibrium theory: a differentiable approach ${ }^{* \dagger}$
}

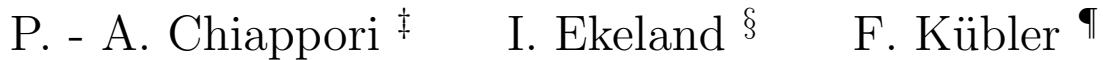 \\ H. M. Polemarchakis ॥
}

Working Paper No. 02-10

Department of Economics, Brown University

April, 2002

\begin{abstract}
Is general equilibrium theory empirically testable? Our perspective on this question differs from the standard, Sonnenschein-DebreuMantel (SDM) viewpoint. While SDM tradition considers aggregate (excess) demand as a function of prices, we assume that what is observable is the equilibrium price vector as a function of the fundamentals
\end{abstract}

*The National Science Foundation provided financial support through the grant SBR 9729559 .

${ }^{\dagger}$ Donald Brown, Gérard Debreu, John Geanakoplos, Fabrizio Germano, Itzhak Gilboa, Roger Guesnerie, Werner Hildenbrand, Rosa Matzkin, Chris Shannon and participants in seminars at the University of Bonn, CentER, the University of Chicago, CORE, the Instituto Veneto during the 1999 Summer School in Economic Theory, at Purdue University during the 1999 NBER conference on General Equilibrium, the University of Paris-I, the University of Tel Aviv and the University of Toulouse made helpful comments.

${ }^{\ddagger}$ Department of Economics, University of Chicago; pchiappo@midway.uchicago.edu

${ }^{\S}$ CEREMADE and Institut de Finance, Université de Paris - IX, Dauphine; ivar.ekeland@dauphine.fr

ฯDepartment of Economics, Stanford University; fkubler@stanford.edu

"Department of Economics, Brown University; herakles_polemarchakis@brown.edu 
of the economy. We apply this perspective to an exchange economy where equilibrium prices and individual endowments are observable. We derive necessary and sufficient conditions that characterize the equilibrium prices, as functions of initial endowments. Furthermore, we show that, if these conditions are satisfied, then the economy can generically be identified. Finally, we show that when only aggregate data are available, observable restrictions vanish. We conclude that the availability of individual data is essential for the derivation of testable consequences of the general equilibrium construct.

Key words: aggregation, equilibrium, identification, testability.

JEL classification numbers: D 10, D 50.

\section{Introduction}

Is general equilibrium theory empirically testable? This question has attracted considerable attention for at least thirty years; that is, at least since the statement of the "Sonnenschein problems": in two seminal papers, Hugo Sonnenschein $(1973,1974)$ posed the question whether the individualistic foundations of general equilibrium theory could generate non-trivial testable restrictions on the aggregate excess demand or market demand functions of an exchange economy. The case of excess demand was solved by Ralf Mantel (1974) and Gerard Debreu (1974); the market demand problem was solved by Jordi Andreu (1983) for finite sets of data, and, recently, by Pierre-André Chiappori and Ivar Ekeland (1999,a) for analytic demand functions. In all cases, the answer is negative, provided there are enough individuals in the economy - a conclusion that confirmed Sonnenschein's intuition and initial arguments.

These (by now classical) results have widely been interpreted as pointing out a severe weakness of general equilibrium theory, namely its inability to generate empirically falsifiable predictions. A prominent illustration of this stand is provided for instance by Kenneth Arrow, who recently listed among the main developments of utility theory the result that "in the aggregate, the hypothesis of rational behavior has in general no implications ", and drew the conclusion that "if agents are different in unspecifiable ways, then [...] very few, if any, inferences can be made" (1991, p. 201). 
Our main claim in the present paper is that this view is overly pessimistic, and that general equilibrium theory can actually generate strong testable predictions, even for large economies. The main idea is in the line of recent contributions by Brown and Matzkin (1996) and Brown and Shannon (2000), and can be summarized as follows. The Sonnenschein-Debreu-Mantel approach concentrates on the properties of excess (or market) demand as a function of prices only. However, this viewpoint is not the only possible one, and actually not the most adequate for assessing the testability of GE theory. ${ }^{1}$ As far as testable predictions are concerned, the structure of aggregate excess demand is not the relevant issue, if only because excess demand is, in principle, not observable, except at equilibrium prices — where, by definition, it vanishes. However, prices are not the only variables that can be observed to vary. Price movements reflect fluctuations of fundamentals, and the relationship between these fundamentals and the resulting equilibrium prices is the natural object for empirical observation. One of the goals of general equilibrium theory is precisely to characterize the properties of this relationship. As it turns out, this characterization generates strong testable restrictions.

We develop our claim in the simple but natural context of an exchange economy, where excess demand depends on both prices and initial endowments. Then equilibrium equations relate equilibrium prices to endowments; the equilibrium manifold is defined as the set of prices and endowments for which excess demand is zero. ${ }^{2}$ We are interested in the local structure of the equilibrium manifold; that is, we study equilibrium prices, locally, as a smooth function of initial endowments. We derive two main results. First, there exist strong restrictions on the local structure of the equilibrium manifold, that can be expressed as a system of partial differential equations, in the spirit of Slutsky relationships. In other words, although utility maximization by individuals does not constrain the shape of excess demand as a function of prices (the SDM conclusion), it does restrict the form of the equilibrium manifold, which is of empirical relevance.

Second, and perhaps more surprisingly, we prove that, if income effects do not vanish, the observation of the equilibrium manifold, as a function of initial endowments, generically identifies the underlying economy, in the

\footnotetext{
${ }^{1}$ There are, of course, deep theoretical reasons for the investigation of the structure of aggregate demand as a function of prices; for instance, the SDM result has strong implications for the convergence of tâ tonnement processes.

${ }^{2}$ See Balasko (1988) for a detailed analysis.
} 
sense that individual preferences can be recovered without ambiguity. In a way, this result is the exact opposite of the SDM conclusion. In the SDM perspective, all the structure due to individual utility maximization is lost by aggregation. Adopting the equilibrium manifold perspective, we reach the opposite conclusion that all the relevant structure is generically preserved, in the sense that the initial economy can (generically) be recovered from the structure of the equilibrium manifold.

These results indicate that the two lines contrasted above - the 'manifold' point of view versus the SDM excess demand approach - generate totally different (and in a sense opposite) conclusions. How can this striking discrepancy be explained? Our interpretation emphasizes a crucial difference: in the manifold approach, individual data (initial endowments) are available, whereas only aggregate variables can be observed in the SDM setting. In other words, we understand our results as suggesting the important conclusion that whenever data are available at the individual level, then utility maximization generates very stringent restrictions upon observed behavior, even if the observed variables (equilibrium prices in our case) are aggregate. From this perspective, whether individual transactions can be observed is irrelevant. Individual determinants of individual choices (such as initial endowments or individual incomes) may do just as well.

A natural question, then, is whether the converse claim also holds: is it the case that, when aggregate variables only are observed, no testable restriction can be generated, at least if the number of individuals is "large enough"? Specifically, assume that only aggregate endowments $\Omega$ can be recorded. These aggregate endowments are redistributed among individuals in the economy according to some rule that is not observed. In particular, fluctuations in $\Omega$ generate changes in individual endowments that are not recorded. What is observed, however, are the corresponding movements of equilibrium prices. In this new context, the equilibrium manifold is observed as a function of aggregate endowments only. Is there any restriction on the form of this relationship?

We show that, under an analyticity condition, when the number of individuals is at least equal to the number of commodities, any (sufficiently smooth) manifold can be (locally) rationalized as the equilibrium manifold of an exchange economy with utility maximizing individuals, for some 'well chosen' redistribution rule. This result closes the argument by confirming that the Walrasian framework cannot generate restrictions on the local structure of the equilibrium manifold when only aggregate data are observable. In this 
sense, although our results emphasize a new aspect of aggregation theory, they remain fully consistent with the conventional wisdom of the field.

Our work is in the line of a former contribution by Brown and Matzkin (1996), who study the restrictions on the structure of the equilibrium manifold from a "non-parametric", revealed preferences perspective. In their paper, Brown and Matzkin derive a set of necessary and sufficient conditions under the form of linear equalities and inequalities that have to be satisfied by any finite data set, and they show that these relationships are indeed restrictive $^{3}$. Our work complements their results in three ways. First, we adopt a differentiable viewpoint, so that our necessary and sufficient conditions take the somewhat more familiar form of a system of partial differential equations, reminiscent of Slutsky conditions. In particular, our conditions can readily be imposed on a parametric estimation of the equilibrium manifold; hence they can be tested using the standard econometric tools used in consumer analysis. ${ }^{4}$ Secondly, the result that these restrictions, if fulfilled, are sufficient to generically recover the underlying economy is original. Thirdly, we extend the analysis to the case where only aggregate endowments are observable, and provide a formal non testability result.

\section{The framework}

\section{$2.1 \quad$ The model}

We consider an exchange economy with $K$ commodities and $N$ individuals. Initial endowments of individual $n$ we denote by $\omega_{n}=\left(\omega_{n}^{1}, \ldots, \omega_{n}^{K}\right) \in \mathbf{R}_{+}^{K}$, and his wealth by $y_{n}=p^{\prime} \omega_{n}=\sum_{k} p_{k} \omega_{n}^{k}$.

Each individual is characterized by a demand function, $x_{n}\left(p, y_{n}\right)$. We assume that demands are derived from the maximization of smooth, strongly quasi-concave utilities; in particular, $x_{n}\left(p, y_{n}\right)$ is smooth and satisfies homogeneity, Walras' law and Slutsky symmetry and negative definiteness.

A smooth map $Z$, defined on $\mathbf{R}_{+}^{K} \times \mathbf{R}_{+}^{K N}$, is an excess demand function

\footnotetext{
${ }^{3}$ This approach has been recently extended by Kübler (2002), Snyder (1999) and Brown and Shannon (2000).

${ }^{4}$ We provide an example of such a parametric analysis in Section 3.
} 
if there exist $N$ individual demand functions $x_{1}, \ldots, x_{N}$, such that

$$
Z(p, \omega)=\sum_{n=1}^{N}\left(x_{n}\left(p, p^{\prime} \omega_{n}\right)-\omega_{n}\right)
$$

If $Z$ is an excess demand function, then it is is homogeneous of degree zero with respect to $p$, and, by Walras' law,

$$
p^{\prime} Z(p, \omega)=0
$$

We use, henceforth, the normalization

$$
p^{\prime} p=1
$$

We denote by $\mathbf{S}^{K-1}$ the unit sphere in $\mathbf{R}^{K}$, and by $\mathbf{S}_{+}^{K-1}$ its intersection with $\mathbf{R}_{+}^{K}$. With the normalization of prices,

$$
Z: \mathbf{S}_{+}^{K-1} \times \mathbf{R}_{+}^{K N} \longrightarrow \mathbf{R}^{K}
$$

and $Z(\bullet, \omega)$ is a map from the unit sphere into $\mathbf{R}^{K}$.

Walras' law implies restrictions on the Jacobian matrix DZ. Differentiating, we get, with obvious notation, that

$$
p^{\prime} D_{p} Z=Z
$$

and, for every commodity,

$$
p^{\prime} D_{\omega_{n}} Z=0 .
$$

Finally, the equilibrium manifold is defined as

$$
\mathcal{E}=\left\{(p, \omega) \in S_{+}^{K-1} \times \mathbf{R}_{+}^{K N} \mid Z(p, \omega)=0\right\} .
$$

In particular, at any point $(p, \omega)$ belonging to $\mathcal{E}$,

$$
p^{\prime} D_{p} Z=0
$$

Taking into account the fact that $Z(\bullet, \omega)$ is defined on the unit sphere, $p^{\prime} p=$ 1 , and that the tangent space at $p$ is precisely $p^{\perp}$, we see that, at every point, $(p, \omega)$ on $\mathcal{E}$, the Jacobian $D_{p} Z$ maps $[p]^{\perp}$ into itself; the same conclusion holds for $D_{\omega_{n}} Z$, for every commodity. 


\subsection{The problem}

Under standard assumptions, the graph of the competitive equilibrium correspondence has the structure of a continuously differentiable manifold. Locally, in a neighborhood, $\mathcal{N}(\bar{p}, \bar{\omega})$, of some arbitrary, non singular point $(\bar{p}, \bar{\omega})$, the equilibrium price can be defined as a function of individual endowments:

$$
(p, \omega) \in \mathcal{E} \cap \mathcal{N}(\bar{p}, \bar{\omega}) \Rightarrow p=\pi(\omega) .
$$

We denote by $\mathcal{N}(\bar{\omega})$ the projection of $\mathcal{N}(\bar{p}, \bar{\omega})$ over $\mathbf{R}_{+}^{K N}$.

Definition 1 A smooth map, $\pi: \mathcal{N}(\bar{\omega}) \mapsto \mathbf{R}_{+}^{K}$, with $\pi^{\prime} \pi=1$, is a regular equilibrium map over $\mathcal{N}(\bar{\omega})$ if there exists a smooth aggregate excess demand function $Z(p, \omega)$, defined on $\mathcal{N}(\bar{p}, \bar{\omega})$, such that $Z(\pi(\omega), \omega)=0$, and $D_{p} Z$ : $[\pi(\omega)]^{\perp} \rightarrow[\pi(\omega)]^{\perp}$ is invertible for all $\omega \in \mathcal{N}(\bar{\omega})$.

Our basic question is thus: What are the conditions for a smooth map, $\pi$, to be a regular equilibrium map over $N(\bar{\omega})$ ?

An immediate remark is that, as pointed out by Balasko (1999), the local nature of the problem is crucial. Indeed, assume that the equilibrium manifold is known globally, including the boundaries of $\mathbf{R}_{+}^{K N}$. Then, one can set $\omega_{2}, \ldots, \omega_{n}$ to zero, so that aggregate excess demand coincides with the excess demand of individual 1; the manifold coincides with inverse demand function of individual 1, which must satisfy well-known restrictions; of course, the same trick can be used for all individuals. The interesting, and more difficult, question we consider refer to the neighborhood of an interior point $(\bar{p}, \bar{\omega})$, where the non-negativity constraints are not binding: $x_{n}^{k}\left(\bar{p}, \bar{p}^{\prime} \bar{\omega}_{n}\right)>0$, for all commodities and individuals.

\section{Characterization of the equilibrium mani- fold}

\subsection{Necessary conditions}

A first result concerns necessary conditions.

Proposition 2 If $\pi$ is a regular equilibrium map over $\mathcal{N}(\bar{\omega})$, then there is an invertible linear map $\Theta(\omega)$ from $[\pi(\omega)]^{\perp}$ into itself, and vectors $\theta_{n}(\omega), 1 \leq$ 
$n \leq N$ in $[\pi(\omega)]^{\perp}$, that depend smoothly on $\omega$, such that, for every $n$, the decomposition

$$
\Theta(\omega) D_{\omega_{n}} \pi(\omega)=I-\theta_{n}(\omega) \pi(\omega)^{\prime}, \quad \omega \in \mathcal{N}(\bar{\omega})
$$

holds. In particular, for any $i$ and $j$, the rank of the matrix $\left(D_{\omega_{j}} \pi-D_{\omega_{i}} \pi\right)$ is at most one. Moreover, the linear map $\Theta(\omega)$ and the vectors $\theta_{n}(\omega)$ are determined uniquely by the map $\pi$. Finally, knowledge of $\Theta(\omega)$ and $\theta_{n}(\omega)$ uniquely identifies the marginal pro- pensity to consume, $a_{n}(p, y)=D_{y} x_{n}(p, y)$, of an individual:

$$
a_{n}\left[\pi(\omega), \pi(\omega)^{\prime} \omega_{n}\right]=\theta_{n}(\omega)+\pi(\omega),
$$

and, for each $n$, the function $a_{n}(p, y)$ satisfies the equations

$$
\left(\frac{\partial a^{k}}{\partial p_{j}}-\frac{\partial a^{j}}{\partial p_{k}}\right) \frac{\partial a^{i}}{\partial y}+\left(\frac{\partial a^{j}}{\partial p_{i}}-\frac{\partial a^{i}}{\partial p_{j}}\right) \frac{\partial a^{k}}{\partial y}+\left(\frac{\partial a^{i}}{\partial p_{k}}-\frac{\partial a^{k}}{\partial p_{i}}\right) \frac{\partial a^{j}}{\partial y}=0
$$

for all commodities $i, j, k$.

Proof See Appendix.

Proposition 1 describes the testable properties of the price function stemming from the underlying, general equilibrium framework. One is the decomposition property (1); is states that there exists a linear map $\Theta$ such that, for any $n$, the matrix $\left(\Theta(\omega) D_{\omega_{n}} \pi(\omega)-I\right)$ is of rank one and vanishes over the subspace $[\pi(\omega)]^{\perp}$. The second set of properties states that the $\theta_{n}$ are related to individual demand functions, hence must satisfy variants of Slutsky relationships. In addition, Proposition 1 shows that the local structure of the manifold allows us to recover the individual income effects - that is, each individual's vector of marginal propensities to consume).

\subsection{An example}

To see how restrictive condition (1) is in general, assume that $K=2$ (then $\pi_{2}$ can be normalized to one) and consider the following functional form for $\pi_{1}$ as a function of the $\omega$ :

$$
\pi_{1}(\omega)=\frac{\sum_{n}\left(A_{n}^{1} \omega_{n}^{1}+A_{n}^{2} \omega_{n}^{2}\right)}{\sum_{n}\left(B_{n}^{1} \omega_{n}^{1}+B_{n}^{2} \omega_{n}^{2}\right)}
$$

with the normalization $A_{1}^{2}+B_{1}^{1}=1$. 
We now derive the restrictions implied by (1) on the coefficients $A_{n}^{i}, B_{n}^{i}$. First,

$$
\frac{\partial \pi_{1}}{\partial \omega_{s}^{k}}-\frac{\partial \pi_{1}}{\partial \omega_{t}^{k}}=\frac{\sum_{j=1}^{2} \sum_{n}\left[\left(A_{s}^{k} B_{n}^{j}-B_{s}^{k} A_{n}^{j}\right)-\left(A_{t}^{k} B_{n}^{j}-B_{t}^{k} A_{n}^{j}\right)\right] \omega_{n}^{j}}{\left[\sum_{n}\left(B_{n}^{1} \omega_{n}^{1}+B_{n}^{2} \omega_{n}^{2}\right) \cdot\right]^{2}}
$$

The decomposition property (1) implies that

$$
\frac{\frac{\partial \pi_{1}}{\partial \omega_{s}^{1}}-\frac{\partial \pi_{1}}{\partial \omega_{t}^{1}}}{\frac{\partial \pi_{1}}{\partial \omega_{s}^{2}}-\frac{\partial \pi_{1}}{\partial \omega_{t}^{2}}}=\pi_{1}
$$

which gives

$$
\frac{\sum_{k} \sum_{n}\left[\left(A_{s}^{1} B_{n}^{k}-B_{s}^{1} A_{n}^{k}\right)-\left(A_{t}^{1} B_{n}^{k}-B_{t}^{1} A_{n}^{k}\right)\right] \omega_{n}^{k}}{\sum_{k} \sum_{n}\left[\left(A_{s}^{2} B_{n}^{k}-B_{s}^{2} A_{n}^{k}\right)-\left(A_{t}^{2} B_{n}^{k}-B_{t}^{2} A_{n}^{k}\right)\right] \omega_{n}^{k}}=\frac{\sum_{n}\left(A_{n}^{1} \omega_{n}^{1}+A_{n}^{2} \omega_{n}^{2}\right)}{\sum_{n}\left(B_{n}^{1} \omega_{n}^{1}+B_{n}^{2} \omega_{n}^{2}\right)} .
$$

This equation must be satisfied for all $\left(\omega_{1}^{1}, \omega_{N}^{K}\right)$. Simple (although tedious) algebra shows that the only form possible for $\pi$ is then:

$$
\pi_{1}(\omega)=\frac{A^{1} \Omega^{1}+\sum_{n} A_{n}^{2} \omega_{n}^{2}}{\sum_{n}\left(1-A_{n}^{2}\right) \omega_{n}^{1}+B^{2} \Omega^{2}} .
$$

The important message of the example is that should an econometric test be based on the relatively flexible functional form (3), then the decomposition condition (1) implies that $A_{s}^{1}=A_{t}^{1}, B_{s}^{2}=B_{t}^{2}$ and $A_{s}^{2}+B_{s}^{1}=1$ for all $s, t-$ that is, a set of strong restrictions.

\subsection{Recovering individual demands}

We now consider the identification problem: to what extent is it possible to recover preferences from the observation of the local structure of the equilibrium manifold? A first remark is that from Proposition 1, the local structure of the equilibrium manifold fully allows to identify individual income effects. We are thus left with a problem in consumer theory, namely: is it possible to recover a demand function $x(p, y)$ from the sole knowledge of its partial derivatives with respect to income, $a(p, y)=D_{y} x(p, y)$ ? We proceed to show that the answer is positive in general. We start with the following restriction:

Assumption 1 : The demand function $x(p, y)$ is such that 
1. the income effect for every commodity $i, \partial x^{i} / \partial y$, is a twice differentiable function of income, and

$$
\frac{\partial^{2} x^{i}}{\partial(y)^{2}} \neq 0
$$

2. there exist at least two commodities $j$ and $k$ such that

$$
\frac{\partial}{\partial y}\left(\ln \frac{\partial^{2} x^{j}}{\partial(y)^{2}}\right) \neq \frac{\partial}{\partial y}\left(\ln \frac{\partial^{2} x^{k}}{\partial(y)^{2}}\right) .
$$

Assumption 1 requires that income effects do not vanish for any commodity, while there are two commodities for which the partial elasticities of the income effects with respect to revenue do not vanish. A few remarks are in order here. First, Assumption 1 implies that there are at least three commodities: $L \geq 3$; a different argument is required for economies with two commodities, $L=2$. Secondly, Assumption 1 rules out specific preferences, such as homothetic or quasi-linear utility functions. Indeed, it can readily be checked that identification is not possible for homothetic utility functions. Intuitively, this is due to the fact that homothetic utilities permit aggregation. In general, however, if demand is non-linear in income, and if income effect do not vanish, Assumption 1 is satisfied for an open and dense set of prices and incomes; which suffices, since continuity then allows for identification. $^{5}$

Finally, it is important to note that Assumption 1 involves only derivatives of the income effects. As such, it can be directly expressed in terms of $a(p, y)$. In particular, from the general perspective of the paper, Assumption 1 can be directly checked from the structure of the manifold, since the latter identifies $a(p, y)$.

The main result is then the following:

Proposition 3 If the demand function, $x(p, y)$, satisfies Assumption 1, then it is uniquely identified by its partial derivatives with respect to income $a(p, y)=$ $D_{y} x(p, y):$ for any demand function, $\xi(p, y)$, if $D_{y} \xi(p, y)=D_{y} x(p, y)$ for all $(p, y)$, then $\xi(p, y)=x(p, y)$, for all $(p, y)$.

Proof See Appendix.

\footnotetext{
${ }^{5}$ This point can be better understood through concept of the "generalized rank" of a demand system, introduced in Lewbel (1991).
} 
Note, in particular, that if $x(p, y)$ satisfies Assumption 1 and $\xi$ is such that $D_{y} \xi(p, y)=D_{y} x(p, y)$ for all $(p, y)$, then $\xi(p, y)$ also satisfies Assumption 1.

We can thus summarize our findings:

Theorem 4 A given smooth map $\pi$ on $\mathcal{N}(\bar{\omega})$ cannot be a regular equilibrium manifold unless it satisfies the testable restrictions given in Proposition 1.

Conversely, if $\pi$ is a regular equilibrium map over $\mathcal{N}(\bar{\omega})$, and if Assumption 1 is satisfied, then the underlying economy, if it exists, is uniquely identified.

\section{The case of aggregate endowments}

\subsection{The problem}

The previous restrictions obtain under a specific hypotheses, namely, that individual endowments are observable. This fact is quite interesting; it suggests, indeed, that testable restrictions require that some data are available at the individual level. In this section, we substantiate this claim by considering the case when aggregate endowments only can be observed. Do restrictions still exist?

Quite obviously, the answer depends on the number of individuals. Take the extreme case of a one individual economy. Then the equilibrium condition boils down to $Z=z_{1}=0$, which means that $\Omega=\omega_{1}$ must be the agent's equilibrium consumption at prices $\pi(\Omega)$. Then $\pi(\Omega)$ is an inverse demand function; as such, it has to satisfy the Slutsky relations, that is, $D_{\omega_{1}} \pi$ must be symmetric on $\omega_{1}^{\perp}$.

This fact is by no means unexpected. With one individual, utility maximization is known to generate restrictive conditions on behavior. What the previous literature suggests, however, is that these conditions might become less and less restrictive as the number of individuals is increased. This intuition turns out to be true, as we now proceed to demonstrate.

\subsection{A formal statement}

Suppose that we can no longer observe the individual endowments $\omega_{n}$, but only the aggregate endowment $\Omega=\sum_{n} \omega_{n}$. Suppose furthermore that, for each value of $\Omega$, this total endowment is distributed across individuals in a 
way which is not observed, and that we only observe some set of equilibrium prices, $p$. What can we predict on the local structure of the mapping $\pi: \Omega \mapsto p$ ? Technically, is it possible, for some arbitrary, smooth $\pi$ satisfying the normalization $\pi^{\prime} \pi=1$, to find $n$ utility functions $U^{1}, \ldots, U^{N}$ and some distribution of endowment $\left(\omega_{1}(\Omega), \ldots, \omega_{N}(\Omega)\right)$, such that the price vector $\pi(\Omega)$ is an equilibrium price for an economy with $N$ individuals, the preference of the $n$-th individual being $U^{n}$ and his endowment $\omega_{n}(\Omega)$ ?

We now answer positively a local version of this problem. Assume that $N \geq K$, and suppose we are given a mapping $\pi: \mathbf{R}^{K} \mapsto S_{+}^{K-1}$. Chose an $\bar{\Omega}$ that satisfies the following, smoothness restriction:

Assumption 2 : There exists an open neighborhood $V(\bar{\Omega})$ of $\bar{\Omega}$ in which the mapping $\Omega \rightarrow(\pi(\Omega), \pi(\Omega) \cdot \Omega)$ is (locally) invertible, and the inverse mapping $A:(p, Y) \rightarrow \Omega$ is analytic in a neighborhood of $(\pi(\bar{\Omega}), \pi(\bar{\Omega}) \cdot \bar{\Omega})$.

Here, $Y=\pi(\Omega) \cdot \Omega$ denotes the economy's total wealth. This assumption deserves a few comments. First, local invertibility does not raise specific problems. It is a standard regularity assumption, that can be expected to hold for almost every $\Omega$. Its main use, here, is to allow to consider the mapping $A$ as a change of variables; that is, any function of $\Omega$ can alternatively be expressed as a function of prices and aggregate income. This technique will be helpful in what follows.

Analyticity is more demanding; it can be viewed as an extreme case of smoothness. However, it is by now known that it is a very useful assumption for this kind of problem (see Chiappori and Ekeland $(1999, b)$.)

\subsection{The main result}

If Assumption 2 is satisfied, then the mapping $A$ has two obvious properties: it is homogenous, and it satisfies $p \cdot A(p, Y)=Y$.

Now, let us just assume that resources are shared equally; the distribution of endowment $\left(\omega_{1}(\Omega), \ldots, \omega_{N}(\Omega)\right)$ is thus defined by

$$
\omega_{n}(\Omega)=\frac{1}{N} \Omega
$$

This implies, in particular, that

$$
\pi(\Omega) \cdot \omega_{n}(\Omega)=\frac{Y}{N}=\frac{\pi(\Omega) \cdot \Omega}{N} .
$$


The problem can be stated as follows: can one find $N$ individual demand functions $x_{1}\left(p, y_{1}\right), \ldots, x_{N}\left(p, y_{N}\right)$, such that

$$
\sum_{n=1}^{N} x_{n}\left(\pi(\Omega), \frac{\pi(\Omega) \cdot \Omega}{N}\right)=\Omega, \quad \Omega \in \mathcal{V}(\bar{\Omega}) .
$$

We can use the change in variables defined by Assumption 2; the previous equation becomes, with obvious notation,

$$
\sum_{n=1}^{N} x_{n}\left(p, \frac{Y}{N}\right)=A(p, Y)
$$

or, using homogeneity

$$
\sum_{n=1}^{N} x_{n}\left(\frac{p}{Y}, \frac{1}{N}\right)=A\left(\frac{p}{Y}, 1\right) .
$$

In words, we are now looking for an economy, the aggregate demand of which is locally equal to some given, analytic function $B\left(\frac{p}{Y}\right)=A\left(\frac{p}{Y}, 1\right)$. The answer is given by a recent result (Chiappori Ekeland 1999,a), which states that this is always possible. Formally,

Theorem 5 Under Assumption 2, and assuming $N \geq K$, there is an open neighborhood $\mathcal{V}$ of $\bar{\Omega}$, and $N$ functions $U_{1}, \ldots, U_{N}$, concave and analytic on $\mathbf{R}^{K}$, such that, for all $\Omega$ in $\mathcal{V}, \pi(\Omega)$ is a system of equilibrium prices for the economy where individual $n$ is characterized by the utility function $U_{n}$ and the endowment

$$
\omega_{n}=\frac{\Omega}{N}
$$

This result confirms the intuition, stated in introduction, that the observation of individual data is necessary to generate testable restrictions. These restrictions reflect both the decentralized nature of the problem and the maximization assumptions made at the individual level; furthermore, they allow, generically, to recover the entire economy. If, on the contrary, only fluctuations in aggregate income can be observed, then no structure is preserved, at least if the number of individuals is large enough. In this sense, albeit our results emphasize a new aspect of aggregation theory, they remain fully consistent with the conventional wisdom of the field ${ }^{6}$.

\footnotetext{
${ }^{6}$ These results can be related to the works by Hildenbrand (1994), who derives testable
} 


\section{Concluding remarks}

A first and obvious conclusion of our work is that the "equilibrium manifold" approach leads to conclusions that differ deeply from the SonnenscheinDebreu-Mantel excess demand perspective. The main conclusion of the latter literature is that all the structure due to individual utility maximization is lost by aggregation. Adopting the equilibrium manifold perspective, we reach the opposite conclusion that all the relevant structure is generically preserved, in the sense that the initial economy can be recovered from the structure of the equilibrium manifold. In that sense, our results both generalize Brown and Matzkin's findings and shed a new light on their scope and status ${ }^{7}$. Also, out interpretation of these results is simple. Rephrasing Arrow's statement quoted in introduction, we believe that in the aggregate, the hypothesis of rational behavior and market equilibrium has in general strong implications even if individuals are different in unspecifiable ways; however, the latter can be tested only insofar as data are available at the individual level. In short, rationality may be testable, but not without individual data ${ }^{8}$.

Finally, what is the empirical relevance of the restrictions derived in the paper? An obvious qualification is that they rely on the impact of changes in individual endowments on aggregate prices. Obviously, the larger the economy, the smaller such effects, and the more difficult it will be to produce empirical work on them. It should be stressed, however, that general equilibrium does not apply only to 'large' economies. On the contrary, the tools

restrictions on the structure of aggregate demand (such as the Law of Demand or the Weak Axiom of Revealed Preference) from assumptions on the form of the distribution of consumptions in the population (increased dispersion, increased spread). The interesting difference is that, while we concentrate upon the properties that are preserved (or destroyed) by aggregation, Hildenbrand's point of view concentrates upon properties that are created by aggregation. A precise assessment of the relationship between the two approaches is a promising line for future investigations.

${ }^{7}$ See also Felix Kübler, Chiappori, Ekeland and Heraklis Polemarchakis (2002) for an extension of these results to the case of uncertainty and incomplete markets.

${ }^{8}$ This conclusion should however be qualified in regard of the remarkable work by Hildenbrand (1994), who derives testable restrictions on the structure of aggregate demand, such as the Law of Demand or the Weak Axiom of Revealed Preferences, from assumptions on the form of the distribution of consumptions in the population — increased dispersion, increased spread. The interesting difference is that, while we concentrate upon the properties that are preserved or destroyed by aggregation, Hildenbrand's point of view concentrates upon properties that are created by aggregation. A precise assessment of the relationships between the two approaches is a promising line for future investigation. 
of general equilibrium theory have been recently applied, in a very successful way, to the analysis of the behavior of 'small' groups. For instance, standard demand theory uses data on households or families, most of which gather several individuals. Models aimed at taking into account the 'non unitary' nature of the interactions at stake usually rely on a 'collective' approach, that postulates only efficiency. With private consumptions - a framework that has been used in most empirical applications - efficient allocations and market equilibria coincide, and GE theory is a relevant tool (see for instance Chiappori and Ekeland 2002). The same approach has also been adopted to the analysis of such groups as committees, clubs, villages and other local organizations, which have also attracted much interest. For instance, many micro studies in development, starting with Robert Townsend's (1994) investigation of risk sharing within an Indian village, are based on data collected at the local level; it is not uncommon to observe endowments (say, individual crops) and prices within the village, a context to which our framework directly applies. Even in large economies, our result may still apply directly when individuals belong to a finite (and "small") number of homogeneous "classes". Finally, an interesting question is how our results can be extended to production economies. The idea is that, in a production context, changes in factor endowments will have an observable impact on factor prices, and that the corresponding equilibrium manifold can in principle be studied in a similar way. All this shall be the subject of further research

\section{References}

1. Andreu, J. (1983), "Rationalization of market demand on finite domain," Journal of Economic Theory, 28, 201-204.

2. Arrow, K. (1991), "Economic Theory and the Hypothesis of Rationality," in The New Palgrave World of Economics, MacMillan, London, 198-210.

3. Balasko, Y. (1988), Foundations of the Theory of General Equilibrium, Academic Press.

4. Brown, D. and R. Matzkin (1996), "Testable Restrictions on the Equilibrium Manifold", Econometrica, Vol. 64, No. 6., pp. 1249-1262. 
5. Brown, D., and C. Shannon (2000), "Uniqueness, stability, and comparative statics in rationalizable Walrasian markets," Econometrica, 68, 1529-1540.

6. Chiappori, P. - A. and I. Ekeland (1999,a), "Aggregation and market de- mand: an exterior differential calculus viewpoint," Econometrica, $67,1435-1458$

7. Chiappori, P. - A. and I. Ekeland (1999,b), "Identifying the economy from the equilibrium manifold: can you recover the invisible hand,"mimeo.

8. Chiappori, P.-A. and I. Ekeland (2002), "The Microeconomics of group Behavior: General Characterization", Mimeo, University of Chicago.

9. Debreu, G. (1974), "Excess demand functions," Journal of Mathematical Economics, 1, 15-21.

10. Hildenbrand, W. (1994), Market Demand, Princeton University Press.

11. Kübler, F. (2002), "Observable restrictions of general equilibrium with financial markets," Journal of Economic Theory, forthcoming.

12. Kübler, F., P. - A. Chiappori, I. Ekeland and H. M. Polemarchakis (2002), "The identification of preferences from equilibrium prices under uncertainty," Journal of Economic Theory, 102, 403-420.

13. Lewbel, A. (1991), "The rank of demand systems," Econometrica, 59, 711-730.

14. Mantel R., (1974) "On the characterization of aggregate excess demand,"

Journal of Economic Theory, 7, 348-353

15. Snyder, S. (1999), "Testable restrictions of Pareto optimal public good provision," Journal of Public Economics, 71, 97-119.

16. Sonnenschein, H. (1973), "Do Walras' identity and continuity characterize the class of community excess demand functions?," Journal of Economic Theory, 6, 345-354.

17. Sonnenschein, H. (1974), "Market excess demand functions," Econometrica, 40, 549-563. 
18. Townsend, R. (1994), "Risk and Insurance in Village India,", Econometrica, 62, 539-591. 


\section{APPENDIX}

\section{Proof of Lemma 1}

From the definition of the excess demand function $Z$, differentiating, we obtain

$$
\frac{\partial Z^{k}}{\partial \omega_{n}^{j}}=\frac{\partial x_{n}^{k}}{\partial y_{n}} p_{j}-\delta_{k}^{j}
$$

where $\delta_{k}^{j}$ is equal to 1 if $j=k$, and equal to 0 otherwise; in matrix notation, this writes

$$
D_{\omega_{n}} Z=\frac{\partial x_{n}}{\partial y_{n}}\left(p, p^{\prime} \omega_{n}\right) p^{\prime}-I .
$$

Differentiating the equation $Z(\pi(\omega), \omega))=0$ with respect to $\omega_{n}$ yields

$$
D_{p} Z D_{\omega_{n}} \pi=-D_{\omega_{n}} Z \text {. }
$$

Replacing in the preceding equation the right-hand side, one gets

$$
D_{p} Z D_{\omega_{n}} \pi=I-\theta_{n}(\omega) \pi(\omega)^{\prime}
$$

where

$$
\theta_{n}(\omega)=\frac{\partial x_{n}}{\partial y}\left[\pi(\omega), \pi(\omega)^{\prime} \omega_{n}\right]
$$

We now split $\mathbf{R}^{K}$ into the orthogonal sum of $[\pi(\omega)]$ and $[\pi(\omega)]^{\perp}$, and express all matrices in some basis $\mathcal{B}=\left\{\pi, b_{2}, \ldots, b_{K}\right\}$, where the vectors $\left\{b_{2}, \ldots, b_{K}\right\}$ all belong to $[\pi(\omega)]^{\perp}$. In this new basis

$$
D_{\omega_{n}} \pi=\left(\begin{array}{cc}
0 & 0 \\
-\Theta(\omega)^{-1} \theta_{n} & \Theta(\omega)^{-1}
\end{array}\right),
$$

where $\Theta(\omega)$ is the $(K-1) \times(K-1)$ matrix, in the (sub)basis $\left\{b_{2}, \ldots, b_{K}\right\}$, of the restriction of $D_{p} Z(\pi(\omega), \omega)$ to $[\pi(\omega)]^{\perp}$; note that, under standard assumptions, $D_{p} Z(\pi(\omega), \omega)$ is of rank $K-1$, hence $\Theta(\omega)$ is invertible.

In particular, the $(K-1) \times(K-1)$ submatrix $\Theta(\omega)^{-1}$ is independent of $n$. A first consequence is that, for any $i$ and $j$, the rank of the matrix $\left(D_{\omega_{j}} \pi-D_{\omega_{i}} \pi\right)$ is at most one; indeed, in the basis $\mathcal{B}$,

$$
\left(D_{\omega_{j}} \pi-D_{\omega_{i}} \pi\right)=\left(\begin{array}{cc}
0 & 0 \\
-\Theta(\omega)^{-1}\left(\theta_{j}-\theta_{i}\right) & 0
\end{array}\right) .
$$


A second consequence, that is actually crucial here, is that the linear map $\Theta(\omega)$ and the vectors $\theta_{n}(\omega)$ above are uniquely defined from $\pi$. Indeed, in the same basis as above, $\Theta(\omega)$ is the inverse of the south-east $(K-1) \times(K-1)$ submatrix of $D_{\omega_{n}} \pi$, while $\theta_{n}(\omega)$ is the product of $-\Theta(\omega)$ by the south-west $(K-1) \times 1$ submatrix of $D_{\omega_{n}} \pi$.

This point can actually be made in a more focused way. Denote by

$$
P(\omega): \mathbf{R}^{K} \mapsto[\pi(\omega)]^{\perp},
$$

sometimes shortened to $P$, the orthogonal projection over $[\pi(\omega)]^{\perp}$. Note that $P(\omega) D_{\omega_{n}} \pi P(\omega)^{\prime}$ is invertible and independent of $n$ :

$$
P(\omega) D_{\omega_{n}} \pi(\omega) P(\omega)^{\prime}=\Theta(\omega)^{-1} .
$$

Then, $\theta_{n}$ is the orthogonal projection of $\left(\partial x_{n} / \partial y\right)$ on $[\pi(\omega)]^{\perp}$, which implies that

$$
\frac{\partial x_{n}}{\partial y}=\theta_{n}+\gamma \pi
$$

where $\gamma$ is some scalar. Since $\pi^{\prime} \pi$ has been normalized to 1 , and since $\pi\left(\partial x_{n} / \partial y\right)=1$, we get $\gamma=1$, and, hence,

$$
\theta_{n}(\omega)=\frac{\partial x_{n}}{\partial y}\left[\pi(\omega), \pi(\omega)^{\prime} \omega_{n}\right]-\pi(\omega)
$$

Finally, we have to check that the knowledge of $\theta_{n}(\omega)$ uniquely defines $\left(\partial x_{n} / \partial y\right)(p, y)$; intuitively, there are "enough degrees of variation" in the $\omega$ to identify the function on the right-hand-side of the equation. A proof is the following. The map $\Phi_{n}: \omega \longmapsto\left(\pi^{\prime}(\omega)^{\prime} \omega_{n}, \pi(\omega)\right)$, which sends $\mathbf{R}_{+}^{K N}$ into $\mathbf{R}_{+}^{K}$. We claim that $D_{\omega_{n}} \Phi_{n}(\omega)$ has rank $K$ for all $\omega$. Indeed, we consider the matrix of partial derivatives with respect to the $\omega_{n}^{k}, k=1, \ldots, K$ :

$$
D_{\omega_{n}} \Phi_{n}(\omega)=\left(\omega_{n}^{\prime} D_{\omega_{n}} \pi(\omega)+\pi^{\prime}(\omega), D_{\omega_{n}} \pi(\omega)\right) .
$$

It maps $\mathbf{R}_{+}^{K}$ into itself. Splitting $\mathbf{R}_{+}^{K}$ into the orthogonal sum of $\pi(\omega)$ and $[\pi(\omega)]^{\perp}$, we get, in the new basis,

$$
D_{\omega_{n}} \Phi_{n}(\omega)=\left(\begin{array}{cc}
1 & 0 \ldots 0 \\
\eta_{n} & \Theta(\omega)^{-1}
\end{array}\right),
$$

where $\eta_{n}$ is some $(K-1)$ vector. Clearly, $D_{\omega_{n}} \Phi_{n}(\omega)$ is of full rank. 
This will allow us, by the implicit functions theorem, to use the following change of variable:

$$
\omega=\left(\omega_{1}, \ldots \omega_{N}\right) \rightarrow\left(\omega_{1}, \ldots, \omega_{n-1}, \pi, y_{n}, \omega_{n+1}, \ldots, \omega_{N}\right) .
$$

The function $\eta_{n}(\omega)$ defined from $\theta_{n}(\omega)$ by

$$
\eta_{n}(\omega)=\theta_{n}(\omega)+\pi(\omega)
$$

In the new coordinate system, it can be written as a function of $\pi$ and $y_{n}$ only, which is the required function.

\section{Proof of Lemma 2}

Since $x(p, y)$ is a demand function, it satisfies Slutsky symmetry:

$$
\frac{\partial x^{k}}{\partial p_{j}}-\frac{\partial x^{j}}{\partial p_{k}}=x^{k} \frac{\partial x^{j}}{\partial y}-x^{j} \frac{\partial x^{k}}{\partial y} .
$$

Differentiating with respect to $y$,

$$
\frac{\partial^{2} x^{k}}{\partial y \partial p_{j}}-\frac{\partial^{2} x^{j}}{\partial y \partial p_{k}}=x^{k} \frac{\partial^{2} x^{j}}{\partial y^{2}}-x^{j} \frac{\partial^{2} x^{k}}{\partial y^{2}}
$$

which can be written as

$$
\frac{\partial a^{k}}{\partial p_{j}}-\frac{\partial a^{j}}{\partial p_{k}}=x^{k} \frac{\partial a^{j}}{\partial y}-x^{j} \frac{\partial a^{k}}{\partial y}, \quad(k, j)_{1}
$$

This provides a system of equations in the $x^{i}$ where all the coefficients are known. Unfortunately, it can readily be checked that this system cannot be of full rank. In fact, the equations are not compatible unless the following condition is fulfilled:

$$
\left(\frac{\partial a^{k}}{\partial p_{j}}-\frac{\partial a^{j}}{\partial p_{k}}\right) \frac{\partial a^{i}}{\partial y}+\left(\frac{\partial a^{j}}{\partial p_{i}}-\frac{\partial a^{i}}{\partial p_{j}}\right) \frac{\partial a^{k}}{\partial y}+\left(\frac{\partial a^{i}}{\partial p_{k}}-\frac{\partial a^{k}}{\partial p_{i}}\right) \frac{\partial a^{j}}{\partial y}=0, \quad(i, j, k) .
$$

If this holds, however, the system is indeterminate, and one further derivation in $y$ is needed. Specifically,

$$
\frac{\partial^{2} a^{k}}{\partial y \partial p_{j}}-\frac{\partial^{2} a^{j}}{\partial y \partial p_{k}}-\left(a^{k} \frac{\partial a^{j}}{\partial y}-a^{j} \frac{\partial a^{k}}{\partial y}\right)=x^{k} \frac{\partial^{2} a^{j}}{\partial y^{2}}-x^{j} \frac{\partial^{2} a^{k}}{\partial y^{2}}, \quad(k, j)_{2} .
$$


From Assumption 1, there exist commodities $j$ and $k$ such that the system consisting of the two equations $(k, j)_{1}$ and $(k, j)_{2}$ in $x^{j}$ and $x^{k}$ is of full rank. This identifies $x^{j}$ and $x^{k}$. Then $(i, k)_{1}$ written for $x^{k}$ and $x^{i}$, allows to identify $x^{i}$.

Finally, a consistency restriction is that the identification gives the same result using $j$ and $i$ instead of $k$ and $i$. This gives

$$
\begin{gathered}
\frac{\partial^{2} a^{k}}{\partial y^{2}} \frac{\partial a^{j}}{\partial y}\left(\left(\frac{\partial a^{i}}{\partial p_{k}}-\frac{\partial a^{k}}{\partial p_{i}}\right) \frac{\partial a^{j}}{\partial y}-\frac{\partial a^{i}}{\partial y}\left(\frac{\partial a^{j}}{\partial p_{k}}-\frac{\partial a^{k}}{\partial p_{j}}\right)\right)= \\
\frac{\partial^{2} a^{j}}{\partial y^{2}} \frac{\partial a^{k}}{\partial y}\left(\left(\frac{\partial a^{j}}{\partial p_{i}}-\frac{\partial a^{i}}{\partial p_{j}}\right) \frac{\partial a^{k}}{\partial y}-\frac{\partial a^{i}}{\partial y}\left(\frac{\partial a^{j}}{\partial p_{k}}-\frac{\partial a^{k}}{\partial p_{j}}\right)\right)+ \\
\frac{\partial a^{j}}{\partial y} \frac{\partial a^{k}}{\partial y}\left(\left(\frac{\partial a^{i}}{\partial p_{k}}-\frac{\partial a^{k}}{\partial p_{i}}\right) \frac{\partial^{2} a^{j}}{\partial y^{2}}-\left(\frac{\partial a^{j}}{\partial p_{i}}-\frac{\partial a^{i}}{\partial p_{j}}\right) \frac{\partial^{2} a^{k}}{\partial y^{2}}\right) .
\end{gathered}
$$

which is a consequence of $(k, j, i)$. 